\title{
Deconvolution of Raman spectra of disordered monolayer graphene: an approach to probe the phonon modes
}

\author{
MD SHERAJUL ISLAM ${ }^{1, *}$ (D, KHALID N ANINDYA ${ }^{1}$, A G BHUIYAN ${ }^{1}$ and A HASHIMOTO ${ }^{2}$ \\ ${ }^{1}$ Department of Electrical and Electronic Engineering, Khulna University of Engineering and Technology, Khulna 9203, \\ Bangladesh \\ ${ }^{2}$ Graduate School of Engineering, University of Fukui, Fukui 910-8507, Japan \\ *Author for correspondence (sheraj_ruet@yahoo.com)
}

MS received 17 November 2018; accepted 28 January 2019; published online 13 May 2019

\begin{abstract}
This paper explores the phonon modes from the Raman spectrum of disordered monolayer epitaxial graphene using the deconvolution technique. The phonon density of states (PDOS) of pristine monolayer graphene has been enumerated and convoluted by a Gaussian function to check the accuracy of the deconvolution process. We show that the original PDOS is recovered by deconvolution of the convoluted spectrum with the same spread function. We propose that the PDOS of pristine monolayer graphene is similar to that of the relative intensity of the deconvoluted Raman spectrum of disordered epitaxial graphene. These results could be used for identifying the intensity of individual phonon modes to justify the structure, and probing the mechanism of changing the phonon modes with various types of defects formed in graphene.
\end{abstract}

Keywords. Graphene; defects; deconvolution; Raman spectra; phonon mode.

\section{Introduction}

Graphene is considered as one of the most exciting materials for next generation nanoelectronics owing to its unique physical properties [1-3]. To characterise and analyse graphene related carbon systems, Raman spectroscopy has become one of the invaluable tools in the last few decades. The Raman spectrum is useful for determining the defect density, detecting the doping levels, finding the layer numbers, quantifying the edge structures, probing the electron-phonon coupling and consequently, the electronic structures [4-13]. All wavelengths of the incident photon become resonant by the zero band gap energy of graphene, and hence, evidence of both structural and electronic properties can be obtained from the Raman spectrum. It is also proven as a prominent method to quantify the selected phonon modes in graphene related systems. Invoking the double resonance processes, Raman spectra exhibit the symmetry-induced optical phonons $[14,15]$. Effects of the phonon can be noticed beyond a photon energy range up to $\sim 350 \mathrm{meV}$ [16]. The study of Raman spectroscopy is therefore of great interest to quantify the solid state properties of graphene.

Although graphene shows extraordinary properties, structural and various crystalline defects are inevitably present during the synthesis process. Impurities, such as B and N, are also intentionally added to modify its electronic structure $[17,18]$. Generally, the graphene Raman spectrum shows four main peaks, usually named as $\mathrm{D}, \mathrm{G}, \mathrm{D}^{\prime}$ and $2 \mathrm{D}$ peaks. Various individual characteristics of graphene are determined by these peaks [19]. The Raman spectrum shows very sharp
$\mathrm{G}$ and 2D peaks for pristine graphene. However, structural defects deconstruct the selection rules that determine the Raman active modes in $\mathrm{sp}^{2}$-bonded graphene related systems. Thus, intriguing Raman characteristics are observed in disordered graphene activating more phonon modes. Though the total intensity of the Raman spectrum is decreased, the features related to various phonon modes are broadened by the defects. Nevertheless, it is very difficult to extract information from the broadened Raman spectra.

Earlier studies on graphite and disordered carbon systems revealed that the Raman spectrum of such structures is interconnected to its phonon density of states (PDOS) $[20,21]$. However, Raman spectra of amorphous carbon are much broader than the PDOS of graphite. Wada et al [22] showed that on convoluting the PDOS of graphite, the shape of broadened PDOS is analogous to the Raman spectra of amorphous carbon. Thus, there may also be a possibility of similitude between the peak positions of the Raman spectra of monolayer disordered graphene and the PDOS of pristine graphene. Wang et al [23] proposed a deconvolution technique to correlate the Raman spectra of disordered carbon with the PDOS of graphite. The deconvolution scheme has the benefit that the deconvoluted spectra are more acute than the main spectra. Hence, it is not required to compare two wide envelopes, rather it is adequate to correlate two curves with more acute peaks. Moreover, this method has the ability to quantitatively demonstrate the changes that occur in the spectra of disordered systems produced using different synthesis techniques. 
(a)

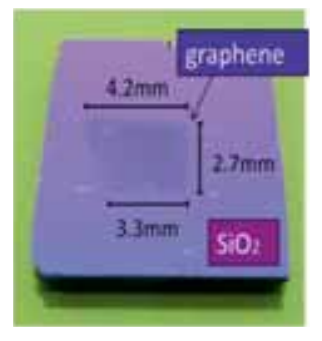

(b)

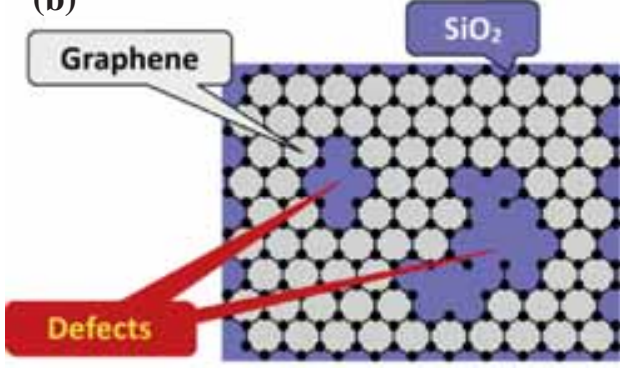

(c)

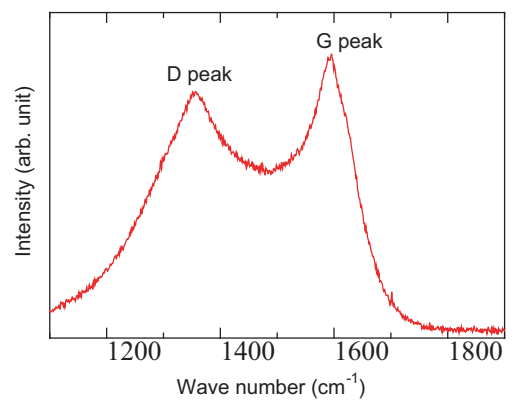

Figure 1. (a) Optical image of the disordered monolayer graphene on $\mathrm{SiO}_{2} / \mathrm{Si}$. (b) Schematic of disordered graphene structure and corresponding (c) Raman spectra. $G$ and D peaks appear at 1590 and $1350 \mathrm{~cm}^{-1}$, respectively.

In this work, deconvolution has been used to extract the phonon modes from the Raman spectra of disordered monolayer epitaxial graphene. We employ the constrained nonlinear method (CNM) [24] to perform deconvolution, which shows a very good precision for the investigation of infrared spectra. We propose that the PDOS of pristine monolayer graphene is similar to that of the relative intensity of the deconvoluted Raman spectra of disordered graphene. The accuracy of the deconvolution technique is also examined by convoluting the PDOS with a Gaussian type spread function. We show that the actual PDOS is recovered by deconvoluting the convoluted spectrum with the same spread function.

\section{Experiments}

The analyses of the present work are performed on a transferred disordered epitaxial graphene as shown in figure 1a. We have used a $4.2 \mathrm{~mm} \times 2.7 \mathrm{~mm}$ monolayer graphene epitaxially grown on silicon carbide by a $\mathrm{Si}$ sublimation technique and transferred to a $\mathrm{SiO}_{2} / \mathrm{Si}$ substrate. During the shifting process, some defects are induced in the graphene sample. The schematic of disordered transferred graphene and the measured Raman spectra are shown in figure $1 \mathrm{~b}$ and c, respectively. A confocal Raman microscope with a spatial resolution of $1 \mu \mathrm{m}$ has been adopted to record the micro-Raman spectra of the sample. We have used a Nd:YVO4-SHG laser equipped with a wavelength of $532 \mathrm{~nm}$ for excitation.

\section{Computational details}

To calculate the phonon modes of graphene, the forced oscillation method (FOM) [25] has been employed, which is very useful for complex and defected crystal systems. The basic concept of FOM is taken from the physical analogy of mechanical resonance. If a lattice dynamical system is exerted by an external force with certain frequency, $\Omega$ for enough time, the response of the system fulfils the resonance condition with a frequency close to the applied one. If a random force given by:

$$
F_{0} \sqrt{M_{l}} \cos \left(\varphi_{l}\right) \cos (\Omega t),
$$

is applied to a lattice dynamical system, the equation of motion of the forced vibration can be represented as:

$$
M_{l} \ddot{u}_{l}+\sum_{l^{\prime}} \phi_{l l^{\prime}} u_{l l^{\prime}}(t)=F_{0} \sqrt{M_{l}} \cos \left(\varphi_{l}\right) \cos (\Omega t),
$$

where $M_{l}$ is the mass of $l$ th atom and $\varphi_{l l^{\prime}}$ is the strength of the spring between the $l$ th and $l^{\prime}$ th atoms. $F_{0}$ is a constant and $\varphi_{l}$ is a random quantity which varies from 0 to $2 \pi$. After sufficient time, $t$, the forced vibration gives the finite energy $E$ of the system. By selecting the appropriate $t$, the density of the states, $g(\Omega)$, is related to the total energy of the system as given by:

$$
g(\Omega)=\frac{8\langle E(\Omega)\rangle}{\pi F_{0}^{2} t N},
$$

where $N$ denotes the number of atoms and $\langle E(\Omega)\rangle$ represents the average total energy of the system which can be calculated numerically.

\section{Results and discussion}

Figure 2a displays the calculated PDOS of pristine monolayer graphene using the FOM. Here, the interactions are considered up to the fourth nearest-neighbour atoms into account. The force constants between the atoms are chosen from the experiment of Gruneis et al [26]. It is well argued that the peaks and valleys of Raman spectra come from the PDOS of the materials. Shuker and Gammon [20] revealed that Raman spectra of disordered systems consist of three components. The first component has three parts, such as the radiation part, the Bose Einstein part and the harmonic oscillation part. The second component includes a wavenumber- dependent matrix element. The third part is the PDOS that provides the spectra, 


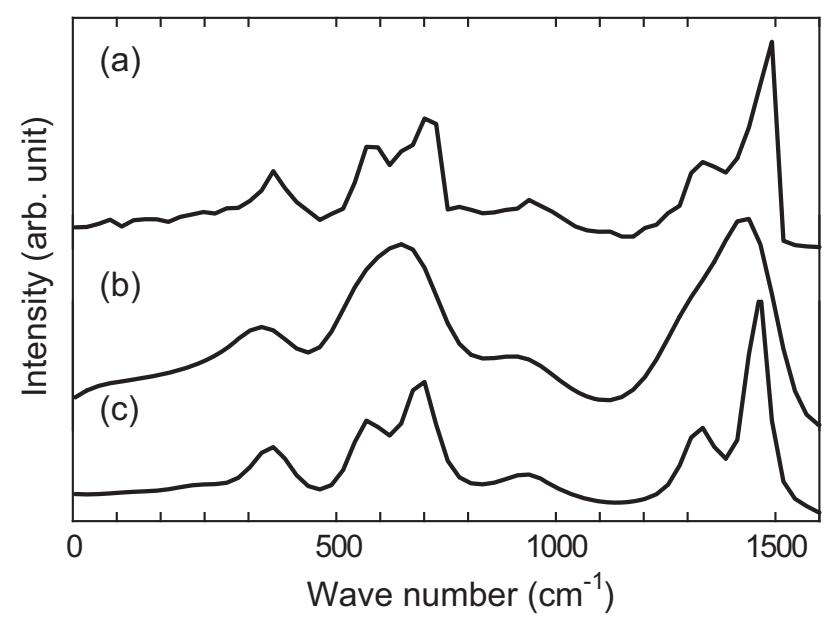

Figure 2. (a) Calculated PDOS of pristine graphene. (b) Convoluted PDOS with a Gaussian type spread function. (c) Deconvoluted PDOS with the same spread function.

combining all the peaks and valleys. It is therefore, generally believed that the PDOS of graphite is similar to the Raman spectra of amorphous carbon. Hence, a comparison may be made between the peak positions of the Raman spectra of monolayer disordered graphene with the calculated PDOS of the pristine system.

The deconvolution technique has been applied to quantitatively explain the changes between the Raman spectra of disordered graphene and the PDOS of pristine graphene. The CNM of deconvolution [24] has been used, which is well developed for the analysis of infrared spectra. The deconvolution technique can be applied to any spectrum if an observed spectrum is related to an ideal spectrum through the convolution with a spread function. Usually, the spread function comes from certain physical processes. It is assumed that the spread function possesses an identical pattern throughout the spectrum. The best estimation of the ideal spectrum has been acquired by the deconvolution of the measured signal and the spread spectrum. The CNM is basically an iterative process modified by Jansson's [24], in which the $k$ th iteration can be written as:

$$
o^{k}=o^{k-1}+r^{k}\left[o^{k-1}\right]\left[i-\left(s * o^{k-1}\right)\right],
$$

with the starting assumption that $o^{1}=i$, where $o$ is the ideal spectrum, $i$ the measured spectrum and $s$ the spread function. $r$ is the relaxation parameter and a function of $o$. The deconvolution results can be obtained in a physically meaningful regime by carefully constructing the relaxation function. Here, the relaxation function $r^{k}\left[o^{k-1}\right]=$ $r_{\max }\left[o^{k-1}\right]^{m}$ is used, where $m$ is a parabolic parameter and $r_{\max }$ is a constant. The parabolic factor, $m$ and relaxation factor, $r_{\max }$ are assumed as 1.5 and $7 \times 10^{18}$, respectively. To perform the deconvolution, we have used the Gaussian function as a spread spectrum. It is well known that the width in the Raman spectra originates from the deviation in the bond angle and bond length of disordered materials and the characteristics of these deviations follow the statistical behaviour. Thus, it should be justified to employ Gaussian as the spread function. Mathematically, the spread function is expressed as:

$$
s(v)=\exp \left(-\frac{\left(v-v_{0}\right)^{2}}{2 \Delta^{2}}\right)
$$

Here, $v_{0}$ is the centre wavenumber and $\Delta$ is the width factor.

To test the accuracy of the calculation, the estimated PDOS of pristine graphene is convoluted by a Gaussian signal. The convoluted result has been deconvoluted with the method mentioned in the earlier section to compare the result with actual PDOS of graphene. Figure 2 shows the PDOS, convoluted PDOS and deconvoluted PDOS of graphene. The results exhibit that the original PDOS is almost extracted after deconvolution of the signal. However, there have been some shifts in the deconvoluted signal from the ideal PDOS spectrum. Several parameters affect the deconvolution process to obtain the actual PDOS. The total number of repetitions and the span of spread function are the most important factors that influence the deconvolution process significantly. To measure the error for different width factors, the deconvoluted result has been further convoluted with the same spread function that has been used for deconvolution. The root mean square (RMS) error has then been calculated for different width factors. The number of iterations also has a vital effect on deconvolution. The deconvoluted spectrum shows more acute peaks when the number of iterations is increased. From our analysis, we find that the number of iterations $\sim 200$ seems appropriate to extract the original PDOS spectrum.

The width factor, $\Delta$ of pristine graphene has been determined by analysing the RMS error value. The PDOS has been convoluted by a Gaussian type spread function having $\Delta$ of $50 \mathrm{~cm}^{-1}$. The obtained signal has then been deconvoluted by the same spread function with the same width factor. Figure 3 shows the dependency of the width factor on the deconvolution spectrum. The calculation is performed considering the width factors $30,50,70$ and $80 \mathrm{~cm}^{-1}$. It is seen from figure 3 that the original PDOS spectrum is not recovered completely, although the same Gaussian function is employed as the convolution process. With the increase in the width factor of the spread function, the peaks in the deconvoluted spectrum become narrower. For a large value of the width factor, the main peaks of the PDOS will disappear. For example, in the case of the width factor of $80 \mathrm{~cm}^{-1}$, the Raman active phonon peak at $1590 \mathrm{~cm}^{-1}$ almost vanished. To determine the appropriate spread function, we have checked the reversibility of the deconvolution process. The deconvoluted PDOS spectrum is further reconvoluted using the spread function with the same width factor as in the case of deconvolution. The RMS difference between these two spectra is then calculated. The RMS error is used to figure out the fluctuations of the deconvolution technique. 


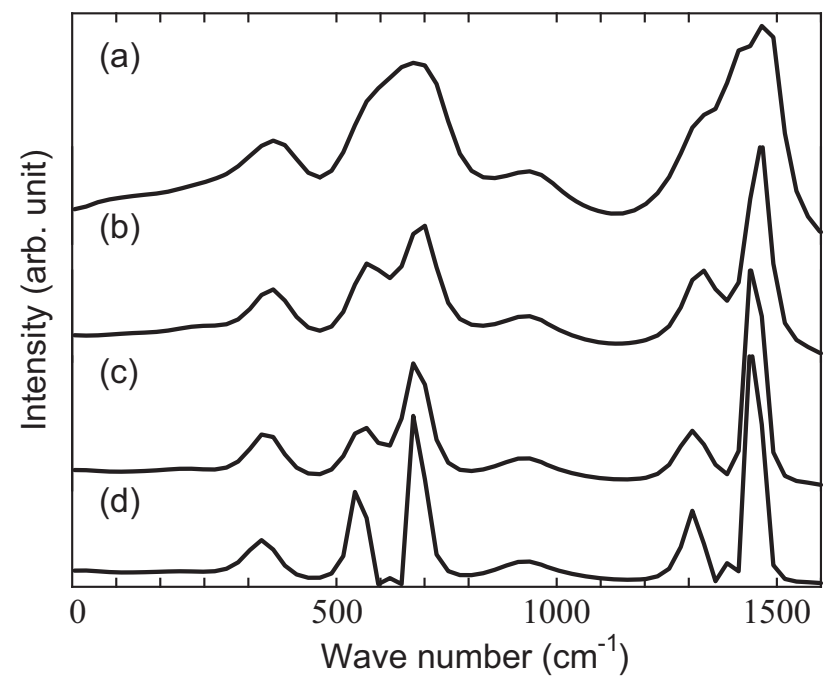

Figure 3. Deconvoluted PDOS spectrum for the spread function with (a) 30 , (b) 50 , (c) 70 and (d) $80 \mathrm{~cm}^{-1}$ width factors.

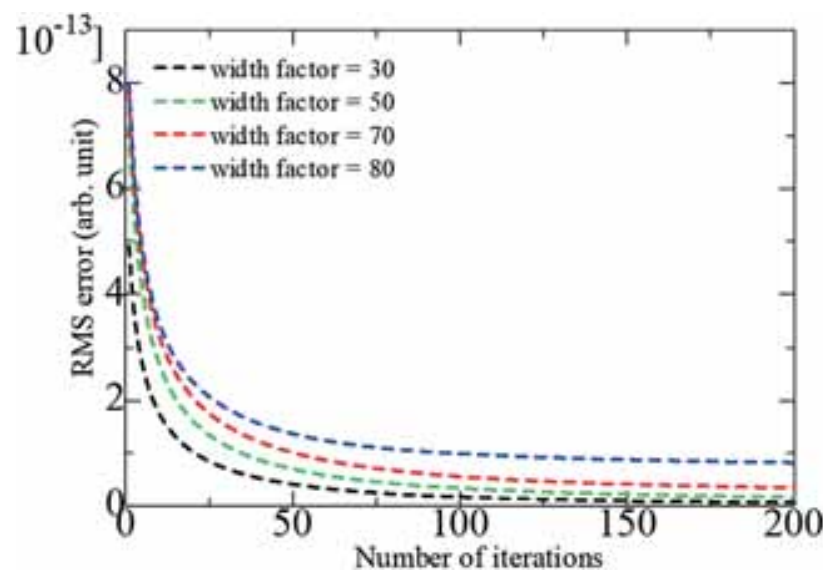

Figure 4. Number of iterations-dependent RMS error of deconvolution with different width factors.

The RMS error is varied with the number of iterations as shown in figure 4. The error diminishes at an exponential rate with the increase in iterations. However, at a particular iteration number, the RMS error is larger for the wider spread function than the narrower spread function. It is distinctly apparent from figure 4 that when we use the width factor of $80 \mathrm{~cm}^{-1}$, the RMS error is higher than that of the width factor of 70,50 or $30 \mathrm{~cm}^{-1}$. Thus, the anomalous spectrum is found for the $80 \mathrm{~cm}^{-1}$ width factor as depicted in figure 3 .

Our next study is focussed on the deconvolution of the Raman spectra of the disordered graphene sample. Generally, the Raman spectrum of graphene exhibits a sharp peak near $1590 \mathrm{~cm}^{-1}$ (G peak) for a pristine sample. The origin of this peak is the high-frequency $E_{2 \mathrm{~g}}$ phonon at $\Gamma$-point of the Brillouin zone [27]. When defects form in a graphene sample, a new peak appears (near $1350 \mathrm{~cm}^{-1}$ ) in the Raman spectra which is absent in pristine graphene. This peak is called the $\mathrm{D}$

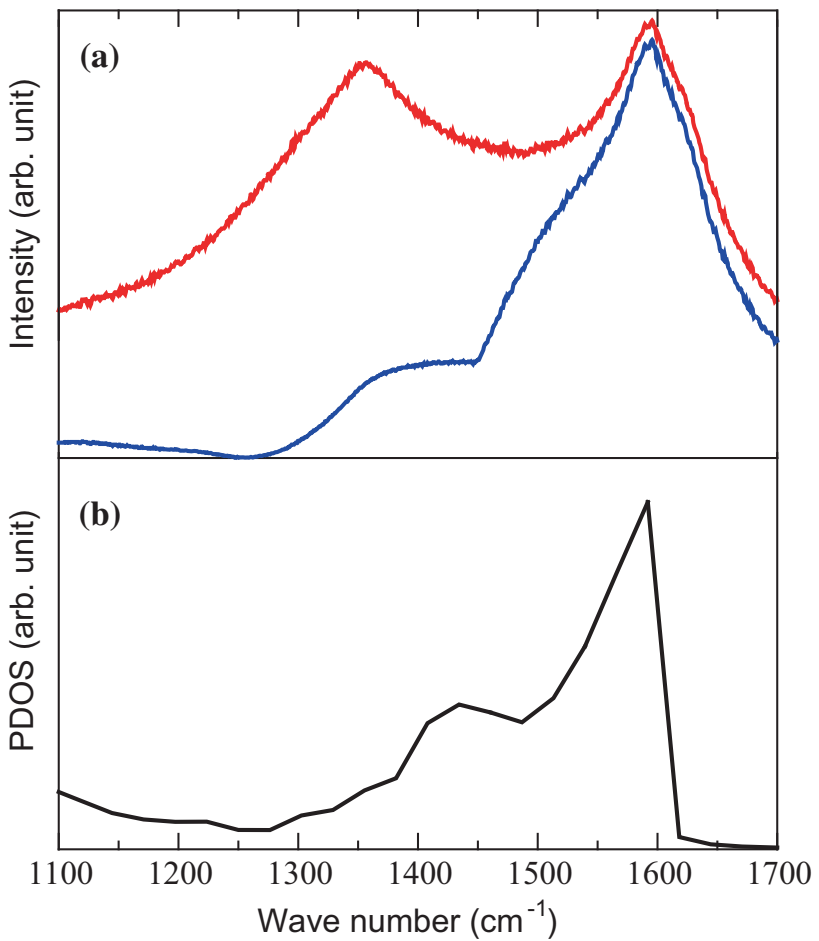

Figure 5. (a) Deconvolution of the Raman spectra of disordered monolayer graphene. The red and blue colours show the typical Raman spectra and deconvoluted spectra, respectively. The width factor and iteration numbers used in the deconvolution process are 70 and $100 \mathrm{~cm}^{-1}$, respectively. (b) Calculated PDOS of pristine graphene in the $1100-1700 \mathrm{~cm}^{-1}$ regime.

peak, which originates from the breathing modes of six-atom rings and is activated by the defects. The double resonance Raman process generates this peak involving inter-valley scattering of the in plane transverse optical phonon near the $\mathrm{K}$ point of the Brillouin zone [28,29]. In figure 5, the red colour shows the Raman spectrum of monolayer transferred epitaxial graphene on $\mathrm{SiO}_{2} / \mathrm{Si}$. Two characteristic peaks such as $\mathrm{G}$ and D peaks are found at 1590 and $1350 \mathrm{~cm}^{-1}$, respectively. The blue line shows the deconvoluted Raman spectra. The calculated PDOS using the FOM is also represented by the black line. As the main characteristic peaks of Raman spectra are found in the range of $1100-1700 \mathrm{~cm}^{-1}$, the PDOS of only these ranges of wave numbers is presented. The deconvoluted Raman spectrum of disordered graphene exhibits the same peak structure and intensity as the PDOS of pristine graphene except the width in the $1370-1480 \mathrm{~cm}^{-1}$ regime. We find that the difference in the width is $<20 \mathrm{~cm}^{-1}$. The similarity between the PDOS and the deconvoluted Raman spectra justifies the usefulness of Shuker's model [20]. Thus, the PDOS of the monolayer pristine system can be recovered from the deconvolution of the Raman spectrum of defective graphene. The deconvolution of the Raman spectrum of disordered graphene can also be employed to detect the intensity of individual phonon modes to study the structural properties 
of the material and to know the process of changing mode due to the various types of defects present in the graphene samples.

\section{Conclusions}

In conclusion, phonon modes of pristine monolayer graphene have been explored from the Raman spectrum of disordered epitaxial graphene using the deconvolution technique. We have calculated the PDOS of pristine monolayer graphene and a convolution has been performed on it by a Gaussian function to examine the deconvolution method. The results reveal that the original PDOS is recovered by deconvolution of the convoluted spectrum with the same spread function. The deconvolution of an actual Raman spectrum of disordered graphene generates a spectrum which is very similar to the PDOS of monolayer pristine graphene. The dissimilarity between the positions of different peaks of the deconvoluted spectrum and the PDOS of graphene are $<20 \mathrm{~cm}^{-1}$. The deconvolution of Raman spectra might be used to compare various types of defective graphene samples, detecting the comparative intensities of different phonon modes to study the structural properties of the sample, and probing the means of variations in different phonon modes due to the various types of defects. The deconvolution of the Raman spectrum could be very valuable characteristic in the analysis of the phonon and properties of graphene related to it.

\section{Acknowledgements}

We are grateful to Prof Satoru Tanaka of Kyushu University, Kyushu, Japan, for providing the sample.

\section{References}

[1] Geim A K and Novoselov K S 2007 Nat. Mater. 6183

[2] Castro Neto A, Guinea F, Peres N M R, Novoselov K S and Geim A K 2009 Rev. Mod. Phys. 81109

[3] Das R K, Saha S, Chelli V R et al 2018 Bull. Mater. Sci. 4186
[4] Das A, Chakraborty B and Sood A K 2008 Bull. Mater. Sci. 31 579

[5] Kalbac M, Reina-Cecco A, Farhat H, Kong J, Kavan L and Dresselhaus M S 2010 ACS Nano 106055

[6] Cançado L G, Silva M G, da Ferreira E H M, Hof F, Kampioti K, Huang K et al 2017 2D Mater. 4025039

[7] Tivanov M S, Kolesov E A, Korolik O V, Saad A M and Komissarov I V 2018 J. Low Temp. Phys. 19020

[8] Lazzeri M, Attaccalite C, Wirtz L and Mauri F 2008 Phys. Rev. B 78081406

[9] Ferrari A C, Meyer C, Scardaci V, Casiraghi C, Lazzeri M, Mauri F et al 2006 Phys. Rev. Lett. 97187401

[10] Ferrari A C 2007 Solid State Commun. 14347

[11] Das A, Pisana S, Chakraborty B, Piscanec S, Saha S K, Waghmare U V et al 2008 Nat. Nanotech. 3210

[12] Islam M S, Bhuiyan A G, Tanaka S, Makino T and Hashimoto A 2014 Appl. Phys. Lett. 105243103

[13] Islam M S, Tamakawa D, Tanaka S, Makino T and Hashimoto A 2014 Carbon 771073

[14] Narula R and Reich S 2008 Phys. Rev. B 78165422

[15] Venezuela P, Lazzeri M and Mauri F 2011 Phys. Rev. B 84 035433

[16] Saito R, Hofmann M, Dresselhaus G, Jorio A and Dresselhaus M S 2011 Adv. Phys. 60413

[17] Islam M S, Ushida K, Tanaka S, Makino T and Hashimoto A 2014 Comput. Mater. Sci. 9435

[18] Fei H, Ye R, Ye G, Gong Y, Peng Z, Fan X et al 2014 ACS Nano 810837

[19] Malard L M, Pimenta M A, Dresselhaus G and Dresselhaus M S 2009 Phys. Rep. 47351

[20] Shuker R and Gammon R W 1970 Phys. Rev. Lett. 25222

[21] Smith J E, Brodsky M H, Crowder B L and Nathan M I 1972 J. Non-Cryst. Solids 8-10 179

[22] Wada N, Gaczi P J and Solin S A 1980 J. Non-Cryst. Solids 35-36 543

[23] Wang Q, Allred D D and Knight L V 1995 J. Raman Spect. 26 1039

[24] Jansson P A 1984 (eds) Deconvolution. With applications in spectroscopy (Orlando: Academic Press)

[25] Williams M L and Maris H J 1985 Phys. Rev. B 314508

[26] Gruneis A, Saito R, Kimura T, Cancado L G, Pimenta M A, Jorio A et al 2002 Phys. Rev. B $\mathbf{6 5} 155405$

[27] Vidano R and Fischbach D B 1978 J. Am. Ceram. Soc. 6113

[28] Thomsen C and Reich S 2000 Phys. Rev. Lett. 855214

[29] Saito R, Jorio A, Souza Filho A G, Dresselhaus G, Dresselhaus M S and Pimenta M A 2001 Phys. Rev. Lett. 88027401 